(C) 2007 IEEE. Reprinted, with permission, from Li Li, A Rank Constrained LMI Algorithm for Decentralized State Feedback Guaranteed Cost Control of Uncertain Systems with Uncertainty Described by Integral Quadratic Constraints , American Control Conference, 2007. ACC '07, July 2007. This material is posted here with permission of the IEEE. Such permission of the IEEE does not in any way imply IEEE endorsement of any of the University of Technology, Sydney's products or services. Internal or personal use of this material is permitted. However, permission to reprint/republish this material for advertising or promotional purposes or for creating new collective works for resale or redistribution must be obtained from the IEEE by writing to pubs-permissions@ieee.org. By choosing to view this document, you agree to all provisions of the copyright laws protecting it 


\title{
A Rank Constrained LMI Algorithm for Decentralized State Feedback Guaranteed Cost Control of Uncertain Systems with Uncertainty Described by Integral Quadratic Constraints
}

\author{
Li Li, Ian R. Petersen
}

\begin{abstract}
The paper presents a numerical algorithm for constructing a decentralized state feedback guaranteed cost controller for an uncertain system. The uncertain systems under consideration contain structured uncertainty described by integral quadratic constraints. The decentralized controller is designed to achieve a closed loop system which is absolutely stable and such that a cost function satisfies a given bound for all admissible uncertainties. The main result gives an algorithm for constructing the desired controller in terms of LMIs subject to rank constraints.
\end{abstract}

\section{INTRODUCTION}

This paper considers the problem of decentralized state feedback control for a class of uncertain systems. The problem of robust decentralized control has attracted a great deal of interest in the control theory literature; e.g., see [1][5]. The results of this paper build on the results in a recent paper [6] which considers a new approach to the problem of decentralized state feedback guaranteed cost control of uncertain systems. A feature of this approach is that it can enable the controller to exploit the interconnections between subsystems. This is achieved by choosing a controller gain matrix which is the diagonal part of a non-decentralized controller gain matrix and the off diagonal terms in the controller gain matrix are treated as uncertainties.

As in [6], we consider a class of uncertain systems with uncertainty described by Integral Quadratic Constraints (IQCs); e.g., see [4]. The specific problem considered in [6] is a guaranteed cost control problem with a given quadratic performance index. A decentralized state feedback controller is constructed such that the corresponding closed loop system is absolutely stable and the cost function satisfies a given bound for all admissible uncertainties.

The design method proposed in [6] involves solving an algebraic Riccati equation of the $H^{\infty}$ control type which is dependent on a number of scaling parameters. Furthermore, some of these scaling parameters are required to satisfy some constraints relating to the norm of the off-diagonal blocks of the controller gain matrix obtained from the solution of the Riccati equation. However, no indication is given in [6] as to how these unknown scaling parameters might be constructed. In this paper, we propose a numerical algorithm which will enable these scaling parameters to be

This work was supported by the Australian Research Council.

School of Information Technology and Electrical Engineering, University of New South Wales at the Australian Defence Force Academy, Canberra ACT 2600, Australia, lleely@itee.adfa.edu.au, irplee.adfa.edu.au. constructed. This numerical algorithm involves the solution to a rank constrained LMI problem, see [7] and the references therein. Although such rank constrained LMI problems may in general be difficult to solve, some of the currently available algorithms, such as LMIRank [8], have been found to lead to solutions to this problem in many practical situations. The paper concludes with an example which illustrates the proposed algorithm.

\section{Problem Statement}

We consider a class of uncertain linear systems of the type considered in the paper [9]. These uncertain systems are described by state equations of the form

$$
\begin{aligned}
\dot{x}(t)= & A x(t)+B_{2} u(t)+\sum_{s=1}^{k} B_{1, s} \xi_{s}(t) ; \\
z_{1}(t)= & C_{1,1} x(t) \\
z_{2}(t)= & C_{1,2} x(t) ; \\
& \vdots \\
z_{k}(t)= & C_{1, k} x(t)
\end{aligned}
$$

where $x(t) \in \mathbf{R}^{n}$ is the state, $u(t) \in \mathbf{R}^{m}$ is the control input, $z_{1}(t) \in \mathbf{R}^{h_{1}}, z_{2}(t) \in \mathbf{R}^{h_{2}}, \ldots, z_{k}(t) \in \mathbf{R}^{h_{k}}$ are the uncertainty outputs and $\xi_{1}(t) \in \mathbf{R}^{r_{1}}, \xi_{2}(t) \in \mathbf{R}^{r_{2}}, \ldots, \xi_{k}(t) \in \mathbf{R}^{r_{k}}$ are the uncertainty inputs. Also, it is assumed that the initial condition $x(0)=x_{0}$ is a Gaussian random vector with mean zero and variance $I$; i.e., $x_{0} \sim N(0, I)$.

It is assumed that the state vector $x(t) \in \mathbf{R}^{n}$ and the control input vector $u(t) \in \mathbf{R}^{m}$ have been decomposed into $p$ components as follows

$$
x=\left[\begin{array}{l}
x_{1} \\
x_{2} \\
\vdots \\
x_{p}
\end{array}\right], \quad u=\left[\begin{array}{l}
u_{1} \\
u_{2} \\
\vdots \\
u_{p}
\end{array}\right],
$$

where $x_{i} \in \mathbf{R}^{n_{i}}, u_{i} \in \mathbf{R}^{m_{i}}$ for $i=1,2, \ldots, p$ and $n=\sum_{i=1}^{p} n_{i}$, $m=\sum_{i=1}^{p} m_{i}$. Each of the components $x_{i}$ is the state vector of a subsystem of the overall uncertain system under consideration, and each of the components $u_{i}$ is regarded as the control input vector for the subsystem with state vector $x_{i}$. Note that no assumptions are made concerning the structure of the system matrices $A$ and $B_{2}$.

Associated with the system (1), is the cost function

$$
J_{0}=\mathbf{E}\left\{\int_{0}^{\infty}\left[x^{\prime}(t) Q x(t)+u^{\prime}(t) R u(t)\right] d t\right\},
$$


where $Q=Q^{\prime}>0, R=R^{\prime}>0$ are given weighting matrices. System Uncertainty. The uncertainty in the above system is described by equations of the form:

$$
\begin{aligned}
\xi_{1}(t)= & \phi_{1}\left(t,\left.x(\cdot)\right|_{0} ^{t},\left.u(\cdot)\right|_{0} ^{t}\right) \\
\xi_{2}(t)= & \phi_{2}\left(t,\left.x(\cdot)\right|_{0} ^{t},\left.u(\cdot)\right|_{0} ^{t}\right) \\
& \vdots \\
\xi_{k}(t)= & \phi_{k}\left(t,\left.x(\cdot)\right|_{0} ^{t},\left.u(\cdot)\right|_{0} ^{t}\right)
\end{aligned}
$$

where the following Integral Quadratic Constraint is satisfied.

Definition 1: (Integral Quadratic Constraint; see [4], [9].) Let $S_{1}>0, S_{2}>0, \ldots, S_{k}>0$, be given positive-definite matrices. Then an uncertainty of the form (4) is an admissible uncertainty for the system (1) if the following conditions hold: Given any locally square integrable control input $u(\cdot)$ and any corresponding solution to equations (1), (4) with an interval of existence $\left(0, t_{\star}\right)$ (that is, $t_{\star}$ is the upper time limit for which the solution exists), then there exists a sequence $\left\{t_{i}\right\}_{i=1}^{\infty}$ such that $t_{i} \rightarrow t_{\star}, t_{i} \geq 0$ and

$$
\int_{0}^{t_{i}}\left(\left\|z_{s}(t)\right\|^{2}-\left\|\xi_{s}(t)\right\|^{2}\right) d t \geq-x^{\prime}(0) S_{s} x(0)
$$

for all $i$ and for $s=1,2, \ldots, k$. Here $\|\cdot\|$ denotes the standard Euclidean norm. Also, note that $t_{\star}$ and $t_{i}$ may be equal to infinity.

In the above definition, the signal $\xi_{i}(\cdot)$ accounts for the presence of uncertain dynamics which are driven by the uncertainty output $z_{i}(\cdot)$. This uncertainty description captures a broad class of uncertainties including nonlinear and timevarying dynamic uncertainties. It also allows to account for effects of non-zero initial conditions of uncertain dynamics as well as noises and delays. The terms on the right hand sides of the IQCs (5) correspond to bounds on these effects. We use the time-domain IQCs to describe the relation between $\xi_{i}(\cdot)$ and $z_{i}(\cdot)$, which is originally from the work of Yakubovich [10]-[12]. This is different from the frequencydomain IQCs introduced in [13], in which the signals under consideration must be square integrable. The time-domain IQC uncertainty description is able to overcome this and allows us to deal with unstable nominal systems; see [4] for more detailed discussions.

We will consider a problem of constructing a decentralized linear state feedback controller of the form

$$
u_{i}(t)=K_{i} x_{i}(t) \quad \forall i=1,2, \ldots, p
$$

such that the resulting closed loop system is absolutely stable and the cost function (3) satisfies a guaranteed cost bound.

Definition 2: The controller (6) is said to be a guaranteed cost controller for the uncertain system (1), (5) with the cost function (3) if the following conditions hold:

(i) The closed loop uncertain system defined by (1), (6) and (5) is internally stable; that is, all of the eigenvalues of $A+B_{2} \tilde{K}$ lie in the open left half plane, where $\tilde{K}=$ $\operatorname{diag}\left(K_{1}, \cdots, K_{p}\right)$.

(ii) There exists a constant $c_{0}>0$ such that the following conditions hold: For any $x_{0} \in \mathbf{R}^{n}$, the closed loop uncertain system defined by (1), (6) and (5) with initial condition $x(0)=x_{0}$ is such that for all admissible uncertainties, the corresponding solution to the closed loop system satisfies

$$
\left[x(\cdot), u(\cdot), \xi_{1}(\cdot), \ldots, \xi_{k}(\cdot)\right] \in \mathbf{L}_{2}[0, \infty)
$$

(hence, $t_{\star}=\infty$ ). Also, the closed loop system is such that the cost (3) satisfies the bound $J_{0} \leq c_{0}$.

Definition 3: (See also [4], [9].) The closed loop uncertain system defined by (1), (6) and (5) is said to be absolutely stable if there exists a constant $c>0$ such that the following conditions hold:

(i) For any initial condition $x(0)=x_{0}$ and any uncertainty inputs $\xi_{s}(\cdot) \in \mathbf{L}_{2}[0, \infty)$, the closed loop uncertain system defined by (1), (6) and (5) has a solution which is defined on $[0, \infty)$.

(ii) Given any admissible uncertainty for the closed loop uncertain system defined by (1), (6) and (5), then all corresponding solutions to the closed loop system satisfy $\left[x(\cdot), \xi_{1}(\cdot), \ldots, \xi_{k}(\cdot)\right] \in \mathbf{L}_{2}[0, \infty)$ (hence, $t_{\star}=\infty$ ) and

$$
\|x(\cdot)\|_{2}^{2}+\sum_{s=1}^{k}\left\|\xi_{s}(\cdot)\right\|_{2}^{2} \leq c\left\|x_{0}\right\|^{2} .
$$

Here, $\|q(\cdot)\|_{2}$ denotes the $\mathbf{L}_{2}[0, \infty)$ norm of the function $q(\cdot)$. That is, $\|q(\cdot)\|_{2}^{2}=\int_{0}^{\infty}\|q(t)\|^{2} d t$.

\section{Controller Design}

A new approach to construct decentralized state feedback guaranteed cost controllers for uncertain systems (1), (5) is presented in [6]. The main idea of [6] is to design a full state feedback controller $u=K x$ using the methodology described in [9]. Then, a decentralized state feedback controller is obtained by taking only the block-diagonal part of the feedback matrix $K$. The ignored blocks of the matrix $K$ are treated as additional uncertainties which are added to the uncertainties in the original uncertain system (1), (5). The readers are referred to [9] for more details. We briefly review this approach in this section.

Let $\bar{m}_{i}=\sum_{k=1}^{i} m_{i}, \tilde{m}_{i}=m-\bar{m}_{i}$ for $i=1,2, \ldots, p, \tilde{k}=k+$ $2(p-1)$ and let $\tau_{1}>0, \ldots, \tau_{\tilde{k}}>0, \beta_{1}^{u}>0, \ldots, \beta_{p-1}^{u}>0, \beta_{1}^{l}>$ $0, \ldots, \beta_{p-1}^{l}>0$ be given constants. We consider the following algebraic Riccati equation

$$
A^{\prime} X+X A+X\left(\tilde{B}_{1} \tilde{B}_{1}^{\prime}-B_{2} E^{-1} B_{2}^{\prime}\right) X+\tilde{C}_{1}^{\prime} \tilde{C}_{1}=0
$$


where

$$
\begin{aligned}
& \tilde{C}_{1}=\left[\begin{array}{c}
Q^{1 / 2} \\
0_{m \times n} \\
\sqrt{\tau_{1}} C_{1,1} \\
\vdots \\
\sqrt{\tau_{k}} C_{1, k} \\
\sqrt{\beta_{1}^{u}} \sqrt{\tau_{k+1}} C_{1,1}^{u} \\
\vdots \\
\sqrt{\beta_{p-1}^{u} \sqrt{\tau_{k+p-1}}} C_{1, p-1}^{u} \\
\sqrt{\beta_{1}^{l}} \sqrt{\tau_{k+p}} C_{1,1}^{l} \\
\vdots \\
\sqrt{\beta_{p-1}^{l}} \sqrt{\tau_{k+2 p-2}} C_{1, p-1}^{l}
\end{array}\right] ; \tilde{D}_{12}=\left[\begin{array}{c}
0_{n \times m} \\
R^{1 / 2} \\
0_{h_{1} \times m} \\
\vdots \\
0_{h_{1} \times m} \\
0_{\tilde{n}_{1} \times m} \\
0_{\tilde{n}_{2} \times m} \\
\vdots \\
0_{\tilde{n}_{p-1} \times m} \\
0_{n_{1} \times m} \\
0_{n_{2} \times m} \\
\vdots \\
0_{n_{p-1} \times m}
\end{array}\right] ; \\
& E=\tilde{D}_{12}^{\prime} \tilde{D}_{12} \\
& \tilde{B}_{1}=\left[\begin{array}{ccc}
\tilde{B}_{1}^{0} & \tilde{B}_{1}^{u} & \tilde{B}_{1}^{l}
\end{array}\right] \text {; } \\
& \tilde{B}_{1}^{0}=\left[\begin{array}{lll}
\frac{1}{\sqrt{\tau_{1}}} B_{1,1} & \cdots & \frac{1}{\sqrt{\tau_{k}}} B_{1, k}
\end{array}\right] \text {; } \\
& \tilde{B}_{1}^{u}=\left[\begin{array}{lll}
\frac{1}{\sqrt{\tau_{k+1}}} B_{2} G_{1}^{u} & \ldots & \frac{1}{\sqrt{\tau_{k+p-1}}} B_{2} G_{p-1}^{u}
\end{array}\right] ; \\
& \tilde{B}_{1}^{l}=\left[\begin{array}{lll}
\frac{1}{\sqrt{\tau_{k+p}}} B_{2} G_{1}^{l} & \cdots & \frac{1}{\sqrt{\tau_{k+2 p-2}}} B_{2} G_{p-1}^{l}
\end{array}\right] ; \\
& C_{1, i}^{u}=\left[\begin{array}{cc}
0_{\tilde{n}_{i} \times \bar{n}_{i}} & I_{\tilde{n}_{i} \times \tilde{n}_{i}}
\end{array}\right], \quad i=1, \cdots, p-1 ; \\
& C_{1,1}^{l}=\left[\begin{array}{ll}
I_{n_{1} \times n_{1}} & 0_{n_{1} \times \tilde{n}_{1}}
\end{array}\right] \text {; } \\
& C_{1, i}^{l}=\left[\begin{array}{lll}
0_{n_{i} \times \bar{n}_{i-1}} & I_{n_{i} \times n_{i}} & 0_{n_{i} \times \tilde{n}_{i}}
\end{array}\right], \quad i=2, \cdots, p-1 ; \\
& G_{1}^{u}=\left[\begin{array}{c}
I_{m_{1} \times m_{1}} \\
0_{\tilde{m}_{1} \times m_{1}}
\end{array}\right] \text {; } \\
& G_{i}^{u}=\left[\begin{array}{l}
0_{\bar{m}_{i-1} \times m_{i}} \\
I_{m_{i} \times m_{i}} \\
0_{\tilde{m}_{i} \times m_{i}}
\end{array}\right], \quad i=2, \cdots, p-1 \text {; } \\
& G_{i}^{l}=\left[\begin{array}{c}
0_{\bar{m}_{i} \times \tilde{m}_{i}} \\
I_{\tilde{m}_{i} \times \tilde{m}_{i}}
\end{array}\right], \quad i=1, \cdots, p-1 .
\end{aligned}
$$

Notice here $\tilde{D}_{12}^{\prime} \tilde{C}_{1}=0$.

The parameters $\tau_{1}>0, \ldots, \tau_{\tilde{k}}>0, \beta_{1}^{u}>0, \ldots, \beta_{p-1}^{u}>$ $0, \beta_{1}^{l}>0, \ldots, \beta_{p-1}^{l}>0$ are to be chosen so that this Riccati equation has a minimal positive-definite solution $X>0$. Let

$$
K=-E^{-1} B_{2}^{\prime} X
$$

and partition $K$ to be compatible with $x, u$ in (2) as follows:

$$
K=\left[\begin{array}{llll}
K_{11} & K_{12} & \ldots & K_{1 p} \\
K_{21} & K_{22} & \ldots & K_{2 p} \\
\vdots & & \ddots & \vdots \\
K_{p 1} & K_{p 2} & \ldots & K_{p p}
\end{array}\right]
$$

We then construct the corresponding decentralized state feedback controller

$$
u(t)=\tilde{K} x(t)
$$

where

$$
\tilde{K}=\operatorname{diag}\left(K_{11}, K_{22}, \ldots, K_{p p}\right) .
$$

Define

$$
\begin{aligned}
& \Delta_{i}^{u}=\left[\begin{array}{llll}
K_{i, i+1} & K_{i, i+2} & \ldots & K_{i p}
\end{array}\right]=G_{i}^{u^{\prime}} K C_{1, i}^{u^{\prime}}, \\
& \Delta_{i}^{l}=\left[\begin{array}{llll}
K_{i+1, i}^{\prime} & K_{i+2, i}^{\prime} & \ldots & K_{p i}^{\prime}
\end{array}\right]^{\prime}=G_{i}^{l^{\prime}} K C_{1, i}^{l^{\prime}} \text {, } \\
& i=1, \cdots, p-1 \text {, }
\end{aligned}
$$

where $G_{i}^{u}, C_{1, i}^{u}, G_{i}^{l}, C_{1, i}^{l}, i=1, \cdots, p-1$ are given in (9).

Theorem 1: Consider the uncertain system (1), (5) with cost function (3). Suppose there exist constants $\tau_{1}>$ $0, \ldots, \tau_{\tilde{k}}>0, \beta_{1}^{u}>0, \ldots, \beta_{p-1}^{u}>0, \beta_{1}^{l}>0, \ldots, \beta_{p-1}^{l}>0$ such that the Riccati equation (8) has a solution $X>0$ and the corresponding full state feedback gain matrix $K$ defined in (10) is such that the sub-matrices defined by (13) satisfy the bounds

$$
\begin{aligned}
& \beta_{i}^{u} \geq\left\|\Delta_{i}^{u}\right\|^{2} \\
& \beta_{i}^{l} \geq\left\|\Delta_{i}^{l}\right\|^{2}, \quad i=1, \cdots, p-1 .
\end{aligned}
$$

Then the corresponding decentralized controller $u(t)=\tilde{K} x(t)$ defined by (11), (12) is a guaranteed cost controller for this uncertain system. Furthermore, the corresponding value of the cost function (3) satisfies the bound

$$
J_{0} \leq \operatorname{tr}\left[X+\sum_{s=1}^{k} \tau_{s} S_{S}\right] .
$$

for all admissible uncertainties and moreover, the closed loop uncertain system is absolutely stable.

Note that the bound in (15) is slightly different from the one derived in [6]. Indeed, in [6] this bound is

$$
\operatorname{tr}\left[X+\sum_{s=1}^{k} \tau_{s} S_{s}+\sum_{s=1}^{p-1} \tau_{k+s} S_{s}^{u}+\sum_{s=1}^{p-1} \tau_{k+p-1+s} S_{s}^{l}\right],
$$

where $S_{s}^{u}, S_{s}^{l}, s=1, \cdots, p-1$ are any positive-definite matrices, thus can be chosen arbitrarily small; therefore the bound in (15) also holds.

Now the problem remaining is that how to solve the optimization problem minimizing the performance bound on the right-hand side of (15) subject to the parameterized Riccati equation (8) and the norm constraints in (14). In the next section, we try to tackle this problem numerically and transform it into an equivalent rank constrained LMI problem, which can be solved by some available software such as LMIRank [8].

\section{A RANK CONSTRAINED LMI APPROACH}

As shown in Theorem 1, the proposed decentralized state feedback controller design involves solving a parameterized game-type Riccati equation. Generally, it is difficult to provide a systematic way to solve such a problem. In this section, we discuss one possible approach to address this difficulty numerically. Similar to the technique used in [14], the idea is to replace Riccati equation (8) and norm constraints (14) with a suboptimal problem involving rank constrained LMIs.

Instead of the Riccati equation (8), consider the following Riccati inequality,

$$
A^{\prime} X+X A+X\left(\tilde{B}_{1} \tilde{B}_{1}^{\prime}-B_{2} E^{-1} B_{2}^{\prime}\right) X+\tilde{C}_{1}^{\prime} \tilde{C}_{1}<0 .
$$


It can be easily proved that Theorem 1 also holds for the controller $\tilde{K}$ in (11), (12) corresponding to the case in which $X$ in (10) is a solution to this Riccati inequality (16) instead of the Riccati equation (8); see [6], [15] for details.

By left and right multiplying (16) with $\bar{X}=X^{-1}$, we obtain

$$
\bar{X} A^{\prime}+A \bar{X}+\left(\tilde{B}_{1} \tilde{B}_{1}^{\prime}-B_{2} E^{-1} B_{2}^{\prime}\right)+\bar{X} \tilde{C}_{1}^{\prime} \tilde{C}_{1} \bar{X}<0 .
$$

Let

$$
\begin{aligned}
\Gamma=\operatorname{diag}\left(I_{n}, I_{m}, \tau_{1} I_{h_{1}}, \cdots, \tau_{k} I_{h_{k}}, \beta_{1}^{u} \tau_{k+1} I_{\tilde{n}_{1}}, \cdots,\right. \\
\left.\beta_{p-1}^{u} \tau_{k+p-1} I_{\tilde{n}_{p-1}}, \beta_{1}^{l} \tau_{k+p} I_{n_{1}}, \cdots, \beta_{p-1}^{l} \tau_{k+2 p-2} I_{n_{p-1}}\right) .
\end{aligned}
$$

Then, by Schur complement and noting that $E=R,(17)$ is equivalent to

$$
\left[\begin{array}{cc}
\bar{X} A^{\prime}+A \bar{X}+\tilde{B}_{1} \tilde{B}_{1}^{\prime}-B_{2} R^{-1} B_{2}^{\prime} & \tilde{X} \tilde{C}_{1}^{\prime} \Gamma^{-\frac{1}{2}} \\
\star & -\Gamma^{-1}
\end{array}\right]<0 .
$$

Define

$$
\begin{aligned}
\bar{\tau}_{i} & =\tau_{i}^{-1}, \quad i=1, \cdots, \tilde{k} \\
\bar{\tau}_{i}^{u} & =\left(\beta_{i}^{u} \tau_{k+i}\right)^{-1}, \quad i=1, \cdots, p-1 \\
\bar{\tau}_{i}^{l} & =\left(\beta_{i}^{l} \tau_{k+p-1+i}\right)^{-1}, \quad i=1, \cdots, p-1
\end{aligned}
$$

Substituting (9) into (18), we have

$$
\left[\begin{array}{cc}
\mathcal{M}_{S} & Q_{s} \\
\star & \mathcal{S}_{s}
\end{array}\right]<0
$$

where

$$
\begin{aligned}
& \mathcal{M}_{s}= \bar{X} A^{\prime}+A \bar{X}-B_{2} R^{-1} B_{2}^{\prime}+\sum_{i=1}^{k} \bar{\tau}_{i} B_{1, i} B_{1, i}^{\prime} \\
&+\sum_{i=1}^{p-1} \bar{\tau}_{k+i} B_{2} G_{i}^{u} G_{i}^{u \prime} B_{2}^{\prime}+\sum_{i=1}^{p-1} \bar{\tau}_{k+p-1+i} B_{2} G_{i}^{l} G_{i}^{l^{\prime}} B_{2}^{\prime} \\
& Q_{S}=\left[\bar{X} Q^{\frac{1}{2}}, 0_{n \times m}, \bar{X} C_{1,1}^{\prime} \cdots \bar{X} C_{1, k}^{\prime},\right. \\
&\left.\bar{X} C_{1,1}^{u^{\prime}} \cdots \bar{X} C_{1, p-1}^{u^{\prime}}, \bar{X} C_{1,1}^{l^{\prime}} \cdots \bar{X} C_{1, p-1}^{l^{\prime}}\right] \\
& \mathcal{S}_{s}=-\operatorname{diag}\left(I_{n}, I_{m}, \bar{\tau}_{1} I_{h_{1}}, \cdots, \bar{\tau}_{k} I_{h_{k}}, \bar{\tau}_{1}^{u} I_{\tilde{n}_{1}}, \cdots, \bar{\tau}_{p-1}^{u} I_{\tilde{n}_{p-1}},\right. \\
&\left.\bar{\tau}_{1}^{l} I_{n_{1}}, \cdots, \bar{\tau}_{p-1}^{l} I_{n_{p-1}}\right) .
\end{aligned}
$$

Next, consider the norm constraints (14). Define $\tau_{i}^{u}=$ $\left(\bar{\tau}_{i}^{u}\right)^{-1}, \tau_{i}^{l}=\left(\bar{\tau}_{i}^{l}\right)^{-1}, i=1, \cdots, p-1$. From (14) and (19), we have

$$
\begin{aligned}
\left\|\Delta_{i}^{u}\right\|^{2} & =\left\|G_{i}^{u^{\prime}} K C_{1, i}^{u^{\prime}}\right\|^{2} \leq \beta_{i}^{u}=\bar{\tau}_{k+i} \tau_{i}^{u}, \quad i=1, \cdots, p-1, \\
\left\|\Delta_{i}^{l}\right\|^{2} & =\left\|G_{i}^{l^{\prime}} K C_{1, i}^{l^{\prime}}\right\|^{2} \leq \beta_{i}^{l}=\bar{\tau}_{k+p-1+i} \tau_{i}^{l}, \quad i=1, \cdots, p-1,
\end{aligned}
$$

which are equivalent to

$$
\begin{array}{r}
{\left[\begin{array}{cc}
\bar{\tau}_{k+i} I_{\tilde{n}_{i}} & C_{1, i}^{u} X B_{2} R^{-1} G_{i}^{u} \\
\star & \tau_{i}^{u} I_{m_{i}}
\end{array}\right] \geq 0, \quad i=1, \cdots, p-1 ;} \\
{\left[\begin{array}{cc}
\bar{\tau}_{k+p-1+i} I_{n_{i}} & C_{1, i}^{l} X B_{2} R^{-1} G_{i}^{l} \\
\star & \tau_{i}^{l} I_{\tilde{m}_{i}}
\end{array}\right] \geq 0, \quad i=1, \cdots, p-1 .}
\end{array}
$$

Furthermore, $\bar{X}=X^{-1}, X>0, \tau_{i}^{u}=\left(\bar{\tau}_{i}^{u}\right)^{-1}, \tau_{i}^{u}>0, \tau_{i}^{l}=$ $\left(\bar{\tau}_{i}^{l}\right)^{-1}, \tau_{i}^{l}>0, i=1, \cdots, p-1$ are equivalent to

$$
\begin{aligned}
& {\left[\begin{array}{ll}
X & I_{n} \\
I_{n} & \bar{X}
\end{array}\right] \geq 0, \quad \operatorname{rank}\left[\begin{array}{cc}
X & I_{n} \\
I_{n} & \bar{X}
\end{array}\right] \leq n} \\
& {\left[\begin{array}{cc}
\tau_{i}^{u} & 1 \\
1 & \bar{\tau}_{i}^{u}
\end{array}\right] \geq 0, \quad \operatorname{rank}\left[\begin{array}{cc}
\tau_{i}^{u} & 1 \\
1 & \bar{\tau}_{i}^{u}
\end{array}\right] \leq 1, \quad i=1, \cdots, p-1}
\end{aligned}
$$

$$
\left[\begin{array}{cc}
\tau_{i}^{l} & 1 \\
1 & \bar{\tau}_{i}^{l}
\end{array}\right] \geq 0, \quad \operatorname{rank}\left[\begin{array}{cc}
\tau_{i}^{l} & 1 \\
1 & \bar{\tau}_{i}^{l}
\end{array}\right] \leq 1, \quad i=1, \cdots, p-1 .
$$

Now consider the performance upper bound on the righthand side of (15). Note that minimizing $\operatorname{tr}\left[X+\sum_{s=1}^{k} \tau_{s} S_{s}\right]$ is equivalent to minimizing $\operatorname{tr}(W)$ where $W \in \mathbf{R}^{n \times n}$ and

$$
\left[\begin{array}{cccc}
W-X & {\left[\begin{array}{lll}
S_{1}^{1 / 2} & \cdots & S_{k}^{1 / 2}
\end{array}\right]} \\
\star & {\left[\begin{array}{ccc}
\bar{\tau}_{1} I_{n} & & \\
& \ddots & \\
& & \bar{\tau}_{k} I_{n}
\end{array}\right]}
\end{array}\right] \geq 0 .
$$

Consider the following optimization problem in the variables $X, \bar{X}, W \in \mathbf{R}^{n \times n}, \bar{\tau}_{i}, i=1, \cdots, \tilde{k}, \tau_{j}^{u}, \bar{\tau}_{j}^{u}, \tau_{j}^{l}, \bar{\tau}_{j}^{l}, \quad j=$ $1, \cdots, p-1$ :

$$
\min \operatorname{tr}(W) \quad \text { subject to (20)-(26). }
$$

Note that this problem is a problem of minimizing a linear cost subject to rank constrained LMIs. As mentioned in the introduction, to solve this problem in our numerical experiments, we use the rank constrained LMI solver LMIRank [8].

We summarize the proposed control design algorithm as follows.

- Solve the rank constrained LMI optimization problem in (27) to obtain $X>0$.

- Calculate $K$ in (10).

- Construct the decentralized state feedback controller $\tilde{K}$ (12).

\section{ILLUSTRATIVE EXAMPLE}

In this section, we consider a problem of decentralized robust guaranteed cost control in order to illustrate the algorithm developed above. We consider an uncertain system described by the following state equations

$$
\dot{x}=A x+B_{1} \Delta C_{1} x+B_{2} u,
$$


where $\Delta=\operatorname{diag}\left(\Delta_{1}, \Delta_{2}, \Delta_{3}\right),\left\|\Delta_{i}\right\| \leq 1$ and

$$
\begin{aligned}
& A=\left[\begin{array}{cccccc}
2 & 2 & 0.5 & 0.5 & 0.4 & 0.2 \\
1 & 1 & 0.5 & 0.5 & 0.4 & 0.2 \\
0 & 0.5 & 2 & 2 & 0 & 0 \\
0.5 & 0 & 2 & 1 & 0.5 & 0 \\
0.1 & 0.1 & 0 & 1 & 3 & 0 \\
0.1 & 0 & 1 & 0 & 1 & 1
\end{array}\right] \\
& B_{2}=\left[\begin{array}{lllll}
1 & 0 & 0 & 0 & 0 \\
1 & 0 & 0 & 0 & 0 \\
0 & 1 & 0 & 0 & 0 \\
0 & 0 & 1 & 0 & 0 \\
0 & 0 & 0 & 0 & 1 \\
0 & 0 & 0 & 1 & 0
\end{array}\right] \\
& B_{1}=\left[B_{1,1}\left|B_{1,2}\right| B_{1,3}\right]=\left[\begin{array}{cc|cc|cc}
0 & 1 & 0.3 & 0.1 & 0.1 & 0.3 \\
0 & 1 & 0.3 & 0.1 & 0.1 & 0.3 \\
1 & 0 & 0 & 0.1 & 0.5 & 0.5 \\
0 & 1 & 0 & 0 & 0 & 0 \\
1 & 0 & 0 & 0.1 & 0 & 0.2 \\
0 & 0 & 0.1 & 0.1 & 0.2 & 0
\end{array}\right] \text {, } \\
& C_{1}=\left[\begin{array}{l}
C_{1,1} \\
\hline C_{1,2} \\
\hline C_{1,3}
\end{array}\right]=\left[\begin{array}{lll}
I_{2} & 0_{2} & 0_{2} \\
\hline 0_{2} & I_{2} & 0_{2} \\
\hline 0_{2} & 0_{2} & I_{2}
\end{array}\right] \text {. }
\end{aligned}
$$

This example is a modification of the example given in [16]. Indeed, the example is modified by the addition of the structured norm bounded uncertainty $\Delta$ and regarding timedelayed states as input uncertainties. In this example, $x, u$ is partitioned such that $\left[n_{1}, n_{2}, n_{3}\right]=[2,2,2],\left[m_{1}, m_{2}, m_{3}\right]=$ $[1,2,2]$. It is desired to construct a decentralized guaranteed cost controller which leads to a guaranteed upper bound on the cost functional

$$
J=\mathbf{E} \int_{0}^{\infty}\left[x^{\prime}(t) Q x(t)+u^{\prime}(t) R u(t)\right] d t,
$$

where

$$
Q=I_{6} ; \quad R=I_{5}
$$

In order to fit this uncertain system into the framework of the theory developed above, we define the uncertainty output variables $z_{i}(t)=C_{1, i} x(t)$ and the uncertainty input variables $\xi_{i}(t)=\Delta z_{i}(t), i=1,2,3$. From the norm bound on $\Delta_{i}$, it follows that the signals $\xi_{i}(t)$ and $z_{i}(t)$ satisfy the IQC (5) for any positive-definite matrices $S_{i}, i=1,2,3$.

We now have an uncertain system of the form (1), (5) and we apply the algorithm presented in Section IV to obtain the optimal values of the parameters $\tau=\left[\tau_{1}, \cdots, \tau_{6}\right], \beta^{u}=$ $\left[\beta_{1}^{u}, \beta_{2}^{u}\right], \beta^{l}=\left[\beta_{1}^{l}, \beta_{2}^{l}\right]$. These optimal parameter values were found to be

$$
\begin{aligned}
\tau & =[8.6826,3.8585,8.3302,73.6211,515.6089 \\
\beta^{u} & =[0.0059,0.0008] \\
\beta^{l} & =[0.0029,0.0008]
\end{aligned}
$$

and the resulting minimum upper bound (15) on the cost was

$$
\operatorname{tr} X=59.3536 \text {. }
$$

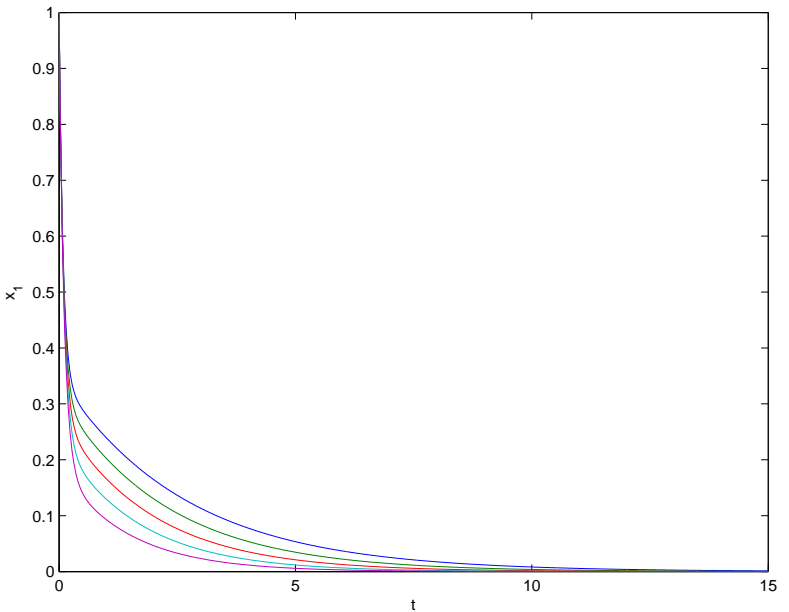

Fig. 1. $\quad x_{1}(t)$ versus $t$ for different values of $\Delta$.

Furthermore, the decentralized guaranteed cost controller was obtained from the block-diagonal components of the full state feedback controller defined in (10) as in (11), (12). The full state feedback controller gain matrix was

$K=\left[\begin{array}{rrrrrr}-10.0118 & -4.4127 & -0.0314 & -0.0417 & -0.0543 & 0.0077 \\ -0.0020 & -0.0295 & -10.4071 & -5.1275 & -0.0091 & -0.0230 \\ -0.0230 & -0.0187 & -5.1275 & -8.5556 & -0.0230 & 0.0091 \\ 0.0390 & -0.0313 & -0.0230 & 0.0091 & -1.3207 & -4.7265 \\ -0.0284 & -0.0259 & -0.0091 & -0.0230 & -11.7011 & -1.3207\end{array}\right]$

and the corresponding decentralized state feedback controller gain matrix was

$$
\tilde{K}=\left[\begin{array}{cccccc}
-10.0118 & -4.4127 & 0 & 0 & 0 & 0 \\
0 & 0 & -10.4071 & -5.1275 & 0 & 0 \\
0 & 0 & -5.1275 & -8.5556 & 0 & 0 \\
0 & 0 & 0 & 0 & -1.3207 & -4.7265 \\
0 & 0 & 0 & 0 & -11.7011 & -1.3207
\end{array}\right] .
$$

This controller was found to yield a stable closed loop nominal system. Furthermore, the initial condition response of the closed loop uncertain system was obtained for different values of $\Delta$ of the form $\Delta=\mu I$ where $\mu=-1,-0.5,0,0.5,1$ respectively. The initial condition was chosen to be $x(0)=$ $\left[\begin{array}{llllll}1 & 0 & 0 & 0 & 0 & 0\end{array}\right]^{\prime}$. The corresponding time response of the state $x_{1}$ for different values of $\Delta$ is shown in Figure 1. Also, the corresponding trajectory of the control input $u_{1}$ is shown in Figures 2.

These results show that the decentralized controller maintains good performance in the face of significant model uncertainty.

\section{CONCLUSIONS}

In this paper, we have presented a numerical algorithm for robust decentralized state feedback guaranteed cost control building on the approach presented in [6]. The key idea of our algorithm is to reformulate the parameterized algebraic Riccati equation in terms of rank constrained LMIs which would be solved to obtain both the matrix $X$ and the scaling parameters.

\section{REFERENCES}

[1] D. D. Siljak, Decentralized Control of Complex Systems. San Diego, CA: Academic Press, 1991. 


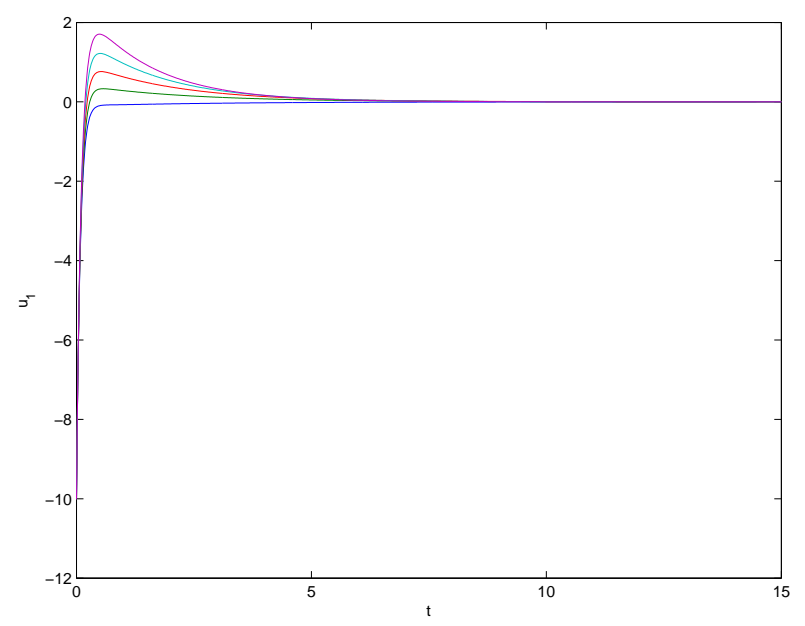

Fig. 2. $u_{1}(t)$ versus $t$ for different values of $\Delta$.

[2] W.-J. Wang and Y. H. Chen, "Decentralized robust control design with insufficient number of controllers," International Journal of Control, vol. 65, pp. 1015-1030, 1996.

[3] G. Zhai, M. Ikeda, and Y. Fujisaki, " $H^{\infty}$ controller design: A matrix inequality approach using a homotopy method," Automatica, vol. 37, no. 4 , pp. 565-572, 2001.

[4] I. R. Petersen, V. Ugrinovski, and A. V. Savkin, Robust Control Design using $H^{\infty}$ Methods. Springer-Verlag London, 2000.

[5] J. Yuz and G. C. Goodwin, "Loop performance assessment for decentralied control of stable linear systems," European Journal of Control, vol. 9, no. 1, pp. 118-132, 2003.

[6] I. R. Petersen, "Decentralized state feedback guaranteed cost control of uncertain systems with uncertainty described by integral quadratic constraints," in American Control Conference, Minneapolis, Minnesota, USA, June 2006, pp. 1333-9.

[7] R. Orsi, U. Helmke, and J. B. Moore, "A Newton-like method for solving rank constrained linear matrix inequalities," Automatica, vol. 42 , no. $11,2006$.

[8] R. Orsi., "LMIRank: software for rank constrained LMI problems, 2005," Available from http://rsise.anu.edu.au/ robert/lmirank/.

[9] A. V. Savkin and I. R. Petersen, "Minimax optimal control of uncertain systems with structured uncertainty," International Journal of Robust and Nonlinear Control, vol. 5, no. 2, pp. 119-137, 1995.

[10] V. A. Yakubovich, "Frequency conditions of absolute stability of control systems with many nonlinearities," Avtomatika i Telemekhanica, vol. 28, no. 6, pp. 5-30, 1967.

[11] _ - "Minimization of quadratic functionals under the quadratic constraints and the necessity of a frequency condition in the quadratic criterion for absolute stability of nonlinear control systems," Soviet Mathematics Doklady, vol. 14, pp. 593-597, 1973.

[12] — - The Methods of Research of Nonlinear Control Systems. Moscow, Russia: Nauka, 1975, ch. 2-3, (in Russian).

[13] A. Megretski and A. Rantzer, "System analysis via integral quadratic constraints," IEEE Transactions on Automatic Control, vol. 42, no. 6, pp. 819-830, 1997.

[14] L. Li, V. A. Ugrinovskii, and R. Orsi, "Decentralized robust control of uncertain Markov jump parameter systems via output feedback," Automatica, to appear.

[15] G. E. Dullerud and F. Paganini, A Course in Robust Control Theory : a Convex Approach. New York: Springer, 2000.

[16] H. S. Wu, "Decentralized stabilizing state feedback controllers for a class of large-scale systems including state delays in the interconnections," Journal of Optimization Theory and Applications, vol. 100, no. 1, pp. 59-87, 1999. 